NBER WORKING PAPER SERIES

\title{
AN EMPIRICAL ASSESSMENT OF ALTERNATIVE MODELS \\ OF RISKY DECISION MAKING
}

\author{
Pamela K. Lattimore \\ Ann D. Witte \\ Joanna R. Baker
}

Working Paper No. 2717

\author{
NATIONAL BUREAU OF ECONOMIC RESEARCH \\ 1050 Massachusetts Avenue \\ Cambridge, MA 02138 \\ September 1988
}

We would like to thank the young adults who agreed to participate in our study and the North Carolina Department of Correction and the Institute for Research in Social Science for allowing us to use subjects in ongoing projects. Jerry Green, Bronwyn Ha11, Bob Lee, John Payne and Helen Tauchen provided valuable comments and suggestions that allowed us to sharpen our thinking and improve our work. Ann Witte's work on this project was supported by a grant from the National Science Foundation (NSF). Points of views expressed do not represent the position of the U.S. Department of Justice, the NSF, or the National Bureau of Economic Research. 
NBER Working Paper \#2717

September 1988

\title{
AN EMPIRICAL ASSESSMENT OF ALTERNATIVE MODELS \\ OF RISKY DECISION MAKING
}

\begin{abstract}
In this paper, we assess the degree to which four of the most commonly used models of risky decision making can explain the choices individuals make when faced with risky prospects. To make this assessment, we use experimental evidence for two random samples of young adults. Using a robust, nonlinear least squares procedure, we estimate a model that is general enough to approximate Kahnemman and Tversky's prospect theory and that for certain parametric values will yield the expected utility model, a subjective expected utility model and a probability-transform model.

We find that the four models considered explain the decision-making behavior of the majority of our subjects. Surprisingly, we find that the choice behavior of the largest number of subjects is consistent with a probability-transform model. Such models have only been developed recently and have not been used in applied settings. We find least support for the expected utility model - - the most widely used model of risky decision making.
\end{abstract}

Pamela $\mathrm{K}$. Lattimore

National Institute of Justice

633 Indiana Avenue, N.W.

Washington, DC 20531

Joanna $\mathbf{R}$. Baker

Department of Management Science

Virginia Polytechnic Institute and State University

Blacksburg, VA 24061
Ann D. Witte

Department of Economics

Wellesley College

Wellesley, MA 02181 


\section{Introduction}

Depending upon one's viewpoint, the modelling of risky decision making is either in a state of chaos or fecundity. ${ }^{I}$ Most applications of this type of decision modeling continue to use the expected utility approach. However, as a result of persistent evidence from laboratory experiments that people do not make decisions in the manner suggested by the expected utility model, theorists have developed a number of alternative representations. When developing these new approaches, researchers have sought models that are generally consistent with the major findings of laboratory studies of choice behavior in risky situations.

Psychologists have generally developed their theories inductively (e.g., Edwards, 1955, Kahnenman and Tuersky, 1979) while economists have pursued deductive approaches (e.g., Handa, 1977; Machina, 1982, Yaari, 1987). This work provides a variety of suggested parametric forms for the preference functional.

To date, as far as we are aware, there have been no attempts to estimate and compare the relative explanatory power of a number of alternative models. In this paper, we begin this task. Specifically, we assess the relative explanatory power of four of the major models of decision making under uncertainty that have been proposed. To do this, we use a functional form that is general enough to approximate Kahnenman and Tversky's prospect theory model and that for certain parametric values will yield the expected utility model, a subjective expected utility model (Savage, 1954; Edwards, 1955), and a model that transforms probabilities but not outcomes (Handa, 1977; Yaari, 1987).

To summarize briefly our most interesting results, we find that the four models explain the decision-making behavior of the majority of our 
subjects. Surprisingly, we find the decision-making behavior of the largest number of subjects to be consistent with a model in which probabilities but not outcomes are transformed. Such models have been developed quite recently and have not been used in applied settings. We find least support for the expected utility model - the most widely used model of risky decision making.

Our results suggest that the decision-making model appropriate for situations involving potential gains is different from the model appropriate for situations with potential losses. This is, of course, a major contention of Kahneman and Tversky (1979) who suggest that the outcome transform will be different in the two settings. Contrary to Kahneman and Tversky's suggestion, we find that it is differences in the way in which probabilities are transformed, not differences in the way outcomes are transformed, that usually distinguishes the model for gains from the model for losses.

The paper is organized as follows. In the next section, we briefly describe the models of risky decision making we consider. Section III presents our empirical model and Sections IV and $V$ describe the data and methods we use to estimate the model. In Section VI, we discuss our results and the final section contains a summary and our conclusions.

\section{The Models Considered}

Consider a simple prospect $Y$ which yields $x_{1}$ with probability $p$ and $x_{2}$ with probability (1-p). Under an expected utility model, the decision maker is assumed to maximize expected utility which is defined as

$$
p u\left(x_{1}\right)+(1-p) u\left(x_{2}\right) .
$$


Note that the decision maker uses objective probabilities to weight the utility, $u\left(x_{i}\right)$, of outcomes. Attitudes toward risk are reflected in the shape of the utility function.

Since the 1950 s some researchers have been uncomfortable with this model because they feel that decision makers do not use objective probabilities, but rather develop subjective probabilities, $s(p)$, that are used to weight outcomes. These subjective probabilities are assumed to follow standard probability rules, but they need not be equal to objective probabilities. For example, Savage (1954) suggests that the individual seeks to maximize subjective expected utility which is defined as

$$
s(p) u\left(x_{1}\right)+s(1-p) u\left(x_{2}\right)
$$

Like the expected utility model this subjective expected utility model assumes that attitudes toward risk are reflected in the utility function. Edwards (1955) develops a similar model but suggests that attitudes toward risk are embedded in the probability transform not in the utility or value function.

More recently a number of economists have developed axiomatic decision models which embed risk attitudes in the probability transform and assume no transformation of outcomes. We refer to such models as probabilitytransform models. As far as we are aware, Handa (1977) developed the first such axiomatic model. Under his model the individual is assumed to maximize

$$
x_{1} h(p)+x_{2} h(l-p)
$$


The function h transforms objective into subjective probabilities and risk: attitudes are reflected in the shape of the probability transform. For prospects with more than two outcomes, Fishburn (1978) has shown that Handa's model implies maximization of expected returns if violations of stochastic dominance are to be avoided.

Quite recently Yaari (1987) has developed a theory dual to the expected utility model that transforms probabilities but not outcomes, embeds risk attitudes in the probability transform and avoids difficulties with previous models of this type (e.g., Handa, 1977; Quiggins, 1982).2 Yaari sees the transform on probabilities as indicating how "perceived rist: is processed into choice" (p.108), Note that Yaari's transform of probabilities is quite different than the transform proposed in subjective expected utility models or Handa's model.

Kahnemari and Tversky (1979) propose an inductively developed theory. "prospect theory," under which both probabilities and outcomes are transformed. Under their model the decision maker is see as maximizing

$$
\pi(\mathrm{p}) \mathrm{v}\left(\mathrm{x}_{1}\right)+\pi(1-\mathrm{p}) \mathrm{v}\left(\mathrm{x}_{2}\right)
$$

where $v$ is a value function which converts outcomes to value and $\pi$ is a decision weighting function over probabilities. The value function is defined on deviations from a reference point. Kahneman and Tversky suggest that the value function is concave above and convex below the reference point and that the function is steeper for losses than for gains. The decision weights are not required to obey the mathematical rules of probability. They measure "the impact of events on the desirability of the prospects and not merely the perceived likelihood of these events" 
(Kahneman and Tversky, 1979, p.285). Conceptually these decision weights are more like Yaari's probability transform than the probability transforms of the other models we have considered. The decision weights are assumed to have the following properties:

1. subadditivity, $\pi(\mathrm{rp})>r \pi(\mathrm{p})$ for $0<\mathrm{r}<1$,

2. subcertainty, $\Sigma \pi\left(p_{i}\right)<1$ and

3. subproportionality, $\pi(p r) / \pi(p)<\pi(p q r) / \pi(p q)$ for $0<p, q, r<1$ which implies that the ratio of the weights of small probabilities is closer to 1 than those of large probabilities.

In Kahneman and Tversky's model, attitudes toward risk are embedded in both the value transform and the decision weighting function.

\section{The Empirical Model}

Consider the following model for a two outcome prospect:

$$
V(Y)=f(p) v\left(x_{1}\right)+f(1-p) v\left(x_{2}\right)
$$

where $V(Y)$ is a preference function over the risky prospect, and $v$ and $f$, respectively, convert outcomes and probabilities into choice relevant variables. As is traditional, we value the risky prospect by its certainty equivalent, $C E$, and assume that the function that transforms outcomes into values also transforms the certainty equivalent of a prospect into a decision relevant value. That is, we assume

$$
V(Y)=v(C E)
$$

Assuming that $\mathrm{v}$ is monotonic and continuous, we obtain

$$
C E=v^{-1}\left[f(p) v\left(x_{1}\right)+f(1-p) v\left(x_{2}\right)\right]
$$


To further specify the model, we must select a functional form for $f$ and $\mathrm{v}$ that will allow us to approximate Kahneman and Tversky's model and that will for certain parameter values yield: (1) an expected utility model, (2) a subjective expected utility model, and (3) a model in which probabilities but not outcomes are transformed.

We assume that $\mathrm{v}$ can be approximated by a power function, e.g. ,

$$
v(x)=x^{\gamma}
$$

The function will be concave, linear or convex as $\gamma \Leftrightarrow 1$. This form has been used extensively in the literature and has been found to provide a good fit to laboratory data (Fishburn and Kochenberger, 1979).

The four models we consider imply different interpretations and values for $\gamma$. The implications are sumarized in Table 1. Under an expected utility model (EU) or a subjective expected utility model (SEU) of the type developed by Savage (1954), one would expect either $\gamma<1$, implying risk aversion, or $\gamma>1$ implying risk-seeking behavior. Under these models a value of $\gamma=1$ would imply risk neutrality and that the decision maker maximizes expected value not expected utility. Under a probabilitytransform model such as that proposed by Handa (1977) or Yaari (1987), one would expect $\gamma=1$. Under a prospect theory model, one would expect that $\gamma$ $<1$ for gains and $\gamma>1$ for losses. To allow for this possibility, we estimate separate models for gains and losses. ${ }^{3}$

For the function that transforms probabilities, we select a form that allows a continuous approximation to Kahneman and Tversky's decision weighting function: 


$$
\mathrm{f}(\mathrm{p})=\frac{\alpha \mathrm{p}^{\beta}}{\alpha \mathrm{p}^{\beta}+(1-\mathrm{p})^{\beta}} .
$$

The properties of $f$ depend upon the values of $\alpha$ and $\beta$, particularly upon whether these parameters are less than, equal to or greater than 1 . If $\alpha<1$ and $\beta<1$, the function is subadditive, subcertain and subproportional as posited by Kahneman and Tversky for their decision weights. The form implied by these parametric values is depicted in panel a of Figure 1 .

Neither Handa (1977) not Yaari (1987) present a specific form for their probability transform. However, Handa does provide us with a picture (1977, Figure 1, p. 113) which suggests that a form like that in panel a would be appropriate.

Subjective expected utility models imply that $f$ is symetric around 0.5 and that people generally overweight probabilities below .5 and underweight probabilities above that value. Further, subjective expected utility models are certain not subcertain. Our function will have the characteristics assumed by subjective expected utility models if $\alpha=1$ and $\beta<1$ as shown in panel b of Figure $1 .^{4}$

Finally, the expected utility model assumes no transformation of probabilities. This implies that $\alpha=\beta=1$. See panel c of Figure 1 .

To obtain our empirical model, we substitute equations (2) and (3) into equation (1) and add a stochastic error term to reflect such things as measurement and judgement error. This yields:

$$
C E=\left[\left(\frac{\alpha p^{\beta}}{\alpha p^{\beta}+(1-p)^{\beta}}\right) x^{\gamma}+\left(\frac{\alpha(1-p)^{\beta}}{p^{\beta}+\alpha(1-p)^{\beta}}\right) x^{\gamma}\right]^{\frac{1}{\gamma}}+\epsilon
$$


which we estimate separately for gains and losses.

If we find that $\alpha<1$ and $\beta<1$ for both gains and losses and that $\gamma<$ 1 for gains and $\gamma>1$ for losses, our work will support a prospect theory model. If, instead, we find the above parameter values for $\alpha$ and $\beta$, but that $\gamma=1$ for both gains and losses than a probability-transform model would seem more appropriate. Our results will support a subjective expected utility model if $\alpha=1, \beta<1$ and $\gamma=1$ and a expected utility mode 1 if $\alpha=\beta=1$ and $\gamma \neq 1$.

\section{The Data}

Our data were obtained from two groups of young adult North Carolinians. The first group of 47 was selected at random from the undergraduate student body at the University of North Carolina at Chapel Hill. These students were part of a computer administered panel study and, thus, were familiar with the type of interactive computer program that we use to administer the decision-making scenarios. Students are the most commonly used subjects for laboratory experiments.

To broaden the socioeconomic representation of participants, we selected a second group of 23 subjects at random from a set of incarcerated, young (18-22) male property offenders. These offenders had been randomly assigned to participate in an innovative job training/ reintegration program.

Each group was presented with two types of risky decisions. The first was a set of standard money gambles (over gains and losses) of the kind generally used in this type of laboratory experiment. The second involved a criminal choice (gain) scenario and a plea bargain (loss) scenario. Responses to the latter type of decisions appeared to be subject to fewer 
attention lapses and judgement errors: perhaps because the scenarios appeared more concrete. We present results only for the second type of risky decisions although our general conclusion would be similar if we used data for the standard money gambles.

Because the amount of time with students was limited, we developed an interactive computer program that contained instructions and presented the decision-making scenarios. Students were paid for their participation in the study.

We provided the offenders in our study with verbal instructions and a set of "practice" risky decisions on the computer because of their lower level of education and lack of experience with interactive computer programs. Their responses to the practice decisions were observed. Misunderstandings were cleared up and questions answered. The offenders were then presented with scenarios by the same interactive computer prograin as used with the students.

To obtain certainty equivalents (CEs) for gains we presented subjects with scenarios like the following:

Suppose that you had decided to break into one of two markets - Jack's or Harry's. Further, suppose that you knew that it would take the same skill to break into either market and that the risk of capture was the same. Further, suppose that you know that Jack has $\$ 900$ in his register half of the time and $\$ 100$ the other half, while Harry always has some cash in his register.

The options available to the subject were then presented in tabular form and the subject was asked "what is the smallest number of dollars there would have to be in Harry's register before you would choose to break into Harry's rather than Jack's?". This is the certainty equivalent value we use when estimating our model. 5 
The possible cash amounts in Jack's register ( $x_{1}$ and $x_{2}$ in our mode1) ranged from $\$ 0$ to $\$ 1000$. The values of $P$ considered were from 0.01 to 0.99 .

The loss scenarios were presented in a similar fashion. Subjects were asked to choose between a certain sentence agreed to in a plea bargain and the risky prospect of going to trial. Possible trial outcomes $\left(x_{1}\right.$ and $\left.x_{2}\right)$ were sentences ranging from 0 to 36 months. 6 values for probabilities were the same as for the illegal-gains scenario. Subjects were asked to indicate the longest sentence length they would accept in a plea bargain in order to forego a trial (the risky prospect).

To prevent the order of presentation of prospects from affecting response ${ }^{7}$, scenario selection was random for both the gain and loss settings. Further, subjects were equally likely to be presented with the gain or loss scenarios first. To prevent some meaningless responses, the subjects were instructed to choose a certainty equivalent value between the two values for the risky prospect (a value between $x_{1}$ and $x_{2}$ ). If a subject choose a value outside this range, they were again instructed to choose a value within the range. This iterative procedure continued until the subject chose a value between $x_{1}$ and $x_{2}$.

Inmates provided certainty equivalents for 34 gain and 34 loss scenarios. Due to the limitation on student time noted above, students provided responses to only 29 gain and 29 loss scenarios. Thus, student models are estimated with 29 data points and inmate models with 34 .

\section{Method of Estimation}

We use an iteratively reweighted nonlinear least squares technique to estimate our model. This procedure is robust to outliers because it gives 
less weight to such observations than would a standard least squares procedure. The method of reweighting observations is due to Beaton and Tukey (1974). We feel it is desirable to downweight outlying observations because for the type of data we are using such observations generally occur due to fatigue and attention lapses. Nonlinear least squares parameter estimates were obtained by a numerical procedure due to Ralston and Jennrich (1978).

\section{Results}

We estimated our decision-making models (gain and loss models) for 57 of the 70 students selected for the study. We were unable to use the data for 13 of our 70 subjects because of unreasonable responses (e.g., same response to all scenarios) or a failure of the parameter estimates to converge. 8

In general our model fit the decision data for our subjects quite well. The $R^{2} s$ for the model ranged from 0.21 to 0.99 for the gain scenarios with $\mathrm{R}^{2}$ s above 0.50 for eighty-two percent of the subjects. For the loss scenarios, the $R^{2}$ s were from 0.13 to 0.98 with $R^{2}$ s above 0.5 for eighty-eight percent of the subjects.

\section{A. The Outcome and Probability Iransforms for Gains}

Consider first the results for the function that transforms outcomes, $v=x^{\gamma}$. For the majority of subjects, over sixty percent (34 of the 57 ), we cannot reject ${ }^{9}$ the null hypothesis that $\gamma=1$. These results would be consistent with either an expected value or probability-transform model. For the bulk of the remaining subjects, thirty percent, our results indicate that $\gamma$ is significantly less than. 1. This implies that for these 
subjects the gains function is concave which would be consistent with an expected utility, subjective expected utility or prospect theory model. For the remaining nine percent of the subjects, our results indicate that $\gamma$ is significantly greater than one. This is not generally expected for any of the models considered, but under an expected utility or subjective expected utility model, this result could be interpreted as indicating risk seeking behavior for this minority group of subjects.

Turning next to the function that transforms probabilities, our results indicate that almost half ( 46 percent) of the subjects transform probabilities in the manner suggested by the subjective expected utility model. Specifically, for these subjects, we cannot reject the null hypothesis that $\alpha=I$ and find support for the alternative hypothesis that $\alpha<1$. See panel b of Figure 1. For nineteen percent of the subjects ( 11 subjects) test statistics support the contention that $\alpha<1$ and $\beta<1$. These parameter values indicate that probabilities are transformed as indicated by prospect theory. See panel a of Figure 1. For sixteen percent of the subjects ( 9 subjects), results indicate that there is no transformation of probabilities (i.e., $\alpha=\beta=1$ ). These results are consistent with either an expected utility or an expected value model.

For the remaining nineteen percent of our subjects (11 subjects), the shape of the probability transform implied by our results were not consistent with any of the models considered. For four of these subjects the test statistics support the contention that $\alpha<1$ and $\beta=1$. The shape of the probability transform implied by these parametric values is depicted in panel a of Figure 2. As can be clearly seen, these subjects underweight all probabilities. Under Handa's model these subjects would be seen as 
globally risk averse and under Yaari's as behaving "pessimistically." For another six of these nonconforming subjects, results indicate that $\alpha>1$ and $\beta<1$. See panel b of Figure 2. These subjects overweight all but high probabilities. The probability transform is supracertain (i.e., $\Sigma f(p)$ $>1$ ) which is not suggested by any of the models we consider. Finally, for one subject, a probability transform like that in panel c of Figure 2 is suggested. Comparing this shape with the shape implied by the subjective expected utility model (panel b of Figure 1), the reader will note that this is the mirror image of the form suggested by that model. This subject underweights probabilities below 0.5 and overweights probabilities above 0.5 .

\section{B. The Outcome and Probability Iransforms for Losses}

For losses, we find that sixty-nine percent of our subjects do not transforms outcomes, i.e., we cannot reject the null hypothesis that $\gamma=1$. For both the gains and loss scenarios, our results for the outcome transform are most in accord with a probability-transform or expected value model. For sixteen of the remaining eighteen subjects, our results indicate risk-seeking behavior. This outcome is predicted by prospect theory. For the two remaining subject test statistics indicated risk aversion over losses.

Turning to results for the function that transforms probabilities, we find that for losses the probability transform is as suggested by prospect theory for 42 percent ( 24 subjects) of our subjects. For these subjects, the probability transform is subadditive for small probabilities, subcertain and subproportional and subjects overweight low probabilities and underweight high ones. See panel a of Figure 1. A probability 
transform of the type suggested by subjective expected utility is support:d for 26 percent of our subjects. See panel b of Figure 1. For sixteen percent of our subjects, results suggest no transform of probabilities as posited by expected utility and expected value models.

As was found for the gains scenarios, the probability transform for a minority of subjects ( 9 subjects) is not suggested by any of the models considered. Results for five of these subjects indicate an overweighting of all but high probabilities. See panel b of figure 2, Results for two subjects are as depicted in panel a of figure 2 and results for one as depicted in panel c. Finally, one subject overweighted all probabilities for the loss scenarios.

\section{Implications for the Decision-Making Model}

Table 2 summarizes our empirical results as they relate to the decision-making models we consider. The first thing to note about these results is that the four types of decision-making models we consider account for the behavior of the majority of the individuals we study. Specifically, for situations offering possible gains, these models best describe the behavior of 70 percent of the subjects and for situations involving losses almost 75 percent of our subjects. Considering the diverse backgrounds of the individual in our study this provides encouraging support for the insightfulness of theorists.

It is interesting that approximately 10 percent of our subjects appear to simply maximize expected value in both gain and loss settings. Another 10 percent of the subjects tend to rather pervasively overweight probabilities. 
Comparing the relative performance of the four models we find that a probability-transform model, a model that transforms probabilities but not outcomes, describes the decision-making behavior of the largest number of subjects. This model well represents the behavior of 42 percent of all subjects when facing gains and 51 percent of all subjects when facing losses. For gains scenarios, the subjective expected utility model is appropriate for the next largest number of subjects (16 percent) and for loss scenarios, a prospect theory model ( 14 percent). It is interesting that the expected utility model appear to describe the behavior of only 5 percent of our subjects.

\section{Conclusions}

We believe that our work makes both a methodological and substantive contribution. From a methodological perspective, we have developed and implemented a procedure that allows researchers to more systematically use laboratory evidence to evaluate alternative models of risky decision making. To date, both economists and psychologists have generally marshalled laboratory evidence, on samples of convenience, to assess or develop a single model of risky decision making. To be more specific, laboratory data are generally obtained from readily available subjects such as college undergraduate volunteers or students in particular classes. The patterns observed in these data are then used either to infer a model (e.g., Kahnenman and Tversky, 1979) or to corroborate a deductively developed model (e.g., Handa, 1977; Yaari, 1987). Corroboration is not generally obtained through the use of standard statistical procedures or standard statistical tests. The relative explanatory power of alternative models is not generally considered. 
We obtain our laboratory data from random samples of subjects, use a mathematical form that encompasses a number of the most commonly used models of risky decision making and assess the relative merits of the alternative models using standard statistical tests. More specifically, our data are for a group that is representative of the undergraduate population of a large state university and a group representative of the young, male property offenders. While these samples are certainly not ideal, they are representative of identifiable populations and come from a broader range of socioeconomic backgrounds than has usually been the case. The mathematical form we use will, under various parametric restrictions, yield a prospect theory, a subjective expected utility, a probability transform and an expected utility model. We estimate the parameters of this model using a robust, nonlinear least squares procedure and use standard statistical tests to distinguish among the alternative models.

Substantively, we provide rather surprising evidence regarding the relative explanatory power of the four models considered. Comfortingly, we find that the four models considered explain the decision-making behavior of the majority (seventy to seventy five percent) of our subjects. Surprisingly, our results provide most support for a probability-transform model. Under this type of model, risk attitudes are reflected in the function that transforms probabilities and outcomes are not transformed. Such models have been developed quite recently (e.g., Handa, 1977; Yaari, 1987) and have not, as far as we are aware, been used in applied settings. However, Yaari ties such models to more widely used models by showing that there is a probability transform model dual to the expected utility model. It is interesting that of the four models we consider, we find least 
support for the expected utility model - the most widely used model of risky decision making.

Turning to insights for future modelling efforts, our results indicate that the decision-making model appropriate for situations involving potential gains is different from the model appropriate for settings involving potential losses. This is, of course, a major contention of Kahneman and Tversky (1979) who suggest that the outcome transform will be different in the two settings. However, we do not find that it is the outcome transform that is different in the two settings. Recall that the majority of our subjects do not appear to transform outcomes. Our results suggest that the major difference in decision making for gain and loss settings results from differences in the way in which probabilities are transformed. Handa (1977) discusses this possibility. For gains, the most common form for the probability transform is that suggested by the subjective expected utility model. This transform indicates that people overweight probabilities below .5 and underweight probabilities below .5 . See panel a of Figure 1. In loss setting, the most common transform is that suggested by prospect theory. With such a transform, people overweight a narrower range of low probabilities and underweight a wider range of high probabilities. See panel b of Figure 1. 
Notes

1. For surveys of the literature, see Schoemaker (1982), Sugden (1986) or Machina (1987).

2. In both Quiggins' (1982) and Yaari's model the transform is not over the simple probability of an outcome but rather over all (Quiggins) or part of the distribution (Yaari, 1987).

3. Work by Kahnenman and Tversky (1979) and Fishburn and Kochenberger (1979) suggests that the reference point is near the current asset level and, thus, separate assessments over gains and losses should capture this aspect of Kahnenman and Tversky's model for the young adult subjects we use. We treat losses as positive numbers as we must if we are to use this outcome transform. This, of course, means that under an expected utility or subjective expected utility model, risk aversion is implied by convexity and risk-seeking behavior by concavity when the model is estimated for losses.

4. Additionally, it should be noted that equation (3) with $\beta=1$ is identical to the decision weighting function suggested by Karmarkar (1978) for his subjectively weighted utility model. Karmarkar suggested that $v(C E)=\frac{\Sigma w_{k} v\left(x_{k}\right)}{\Sigma w_{k}}$, where $w_{k}=\frac{p^{\alpha}}{p^{\alpha}+(1-p)^{\alpha}}$. For prospects involving more than two outgcomes, our decision weighting function would be $\pi-\frac{\alpha \mathrm{p}_{\mathrm{k}}}{\alpha \mathrm{p}_{\mathrm{k}}^{\beta}+\sum_{\mathrm{j}=1}^{\mathrm{p}} \mathrm{p}_{\mathrm{j}}^{\beta}}, \mathrm{j} \neq \mathrm{k}$.

5. The careful reader will note that this is only a very close approximation to a true certainty equivalent. However, this method of presentation greatly simplified the subjects' task and increased their understanding of the decision making problem. Given rounding this "threshold equivalent" should be virtually identical to the certainty equivalent. 
6. These sentence lengths are reasonable under North Carolina statutes. See Clarke and Rubinsky (1981).

7. See Tversky and Kahneman (1982) for a discussion.

8. For gain scenarios, seven subjects provided unreasonable responses and parameter estimates for six subjects failed to converge. For loss scenarios, eleven subjects provided unreasonable responses and parameter estimates for two subjects failed to converge.

9. Unless otherwise noted all two-tailed tests of significance are at the $\alpha=.05$ level and all one-tailed tests at the $\alpha=.025$ level. 


\section{REFERENCES}

Beaton, A.E. and J.W. Tukey (1974). "The fitting of power series, meaning polynomials, illustrated on band-spectroscopic data." Technometrics 16: pp. 147-185.

Clarke, S.H. and E.W. Rubinsky (1981). "North Carolina's Fair Sentencing Act: Explanation, Text, and Felony Classification Table." Chapel Hill, NC: Institute of Government, University of North Carolina.

Fishburn, P. and G.A. Kochenberger (1979). "Two-piece von Neumann-Morgenstern utility functions." Decision Sciences 10: pp. $503-518$.

Edwards, W. (1955). "The predictior of decisions among bets." Journal of Experimental Psychology 50: PF. 201-214.

Handa, J, (1977). "Risk, probability, and a new theory of cardinal utility." Journal of Political Economy 85: pp. 97-122.

Kahneman, D. and A. Tversky (1979). "Prospect theory: an analysis of decision under risk." Econometrica 47: pp. 263-291.

Karmarkar, U.S. (1978). "Subjectively weighted utility: a descriptive extension of the expected utility model." Organizational Behavior and Human Performance 24: pp. 67-72.

Machina, M. (1982). "'Expected utility' analysis without the independence axiom." Econometrica 50: pp. 277-323.

Machina, M. (1987). "Choice under uncertainty: problems solved and unsolved." Journal of Economic Perspectives 1: pp. 121-154.

Quiggin, J. (1982). "A theory of anticipated utility." Journal of Economic Behavior and Organization 3: pp. 323-343.

Ralston, M.L. and R.I. Jennrich (1978). "Dud, a derivative-free algorithm for nonlinear least squares." Technometrics 20: pp. 7-14.

Savage, L.J. (1954). The Foundations of Statistics. New York: Wiley.

Schoemaker, P.J.H. (1982). "The expected utility model: Its variants, purposes, evidence, and limitations". Journal of Economic Literature 20: pp. $529-563$.

Sugden, R. (1986). "New developments in the theory of choice under uncertainty." Bulletin of Economic Research 38: pp. 1-24.

Tversky, A. and D. Kahneman (1982) "Judgement under Uncertainty:

Heuristics and Biases" in D. Kahneman, P. Slovic and A. Tverksy, eds., Judgement under Uncertainty: Heuristics and Biases. Cambridge: Cambridge Univ. Press, pp. 3-20.

Yaari, M.E. (1987). "The dual theory of choice under risk." Econometrica 55: pp. 95-115. 

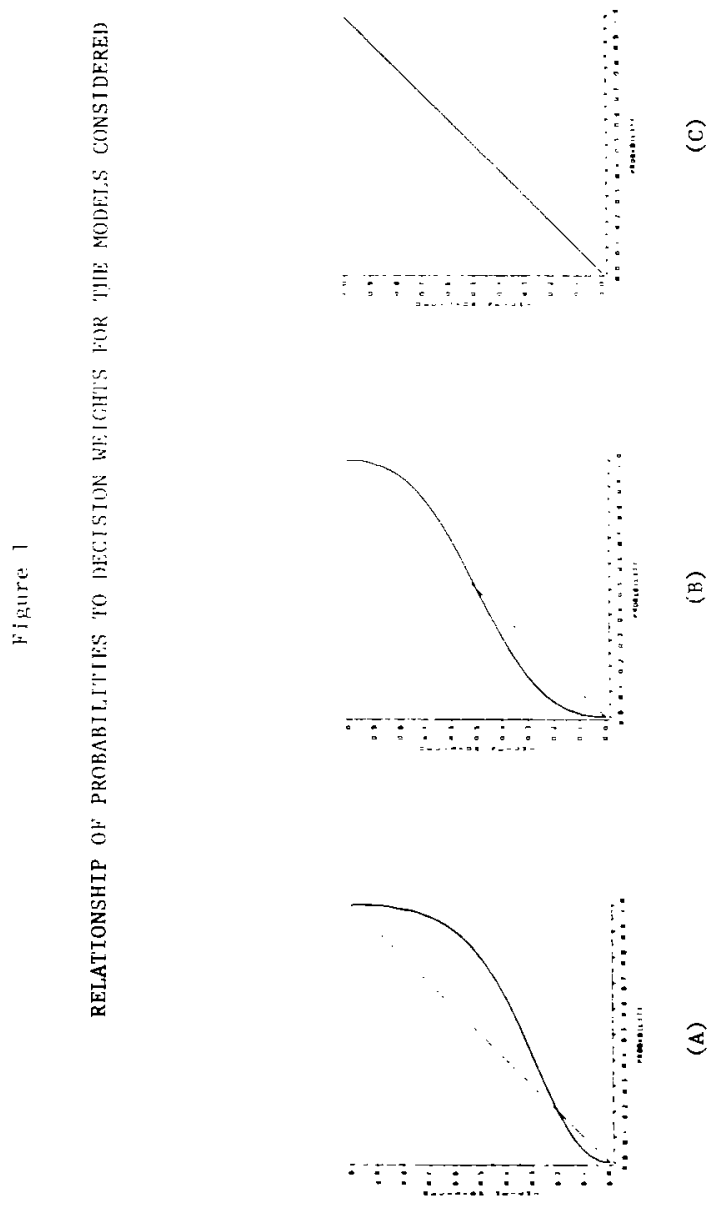

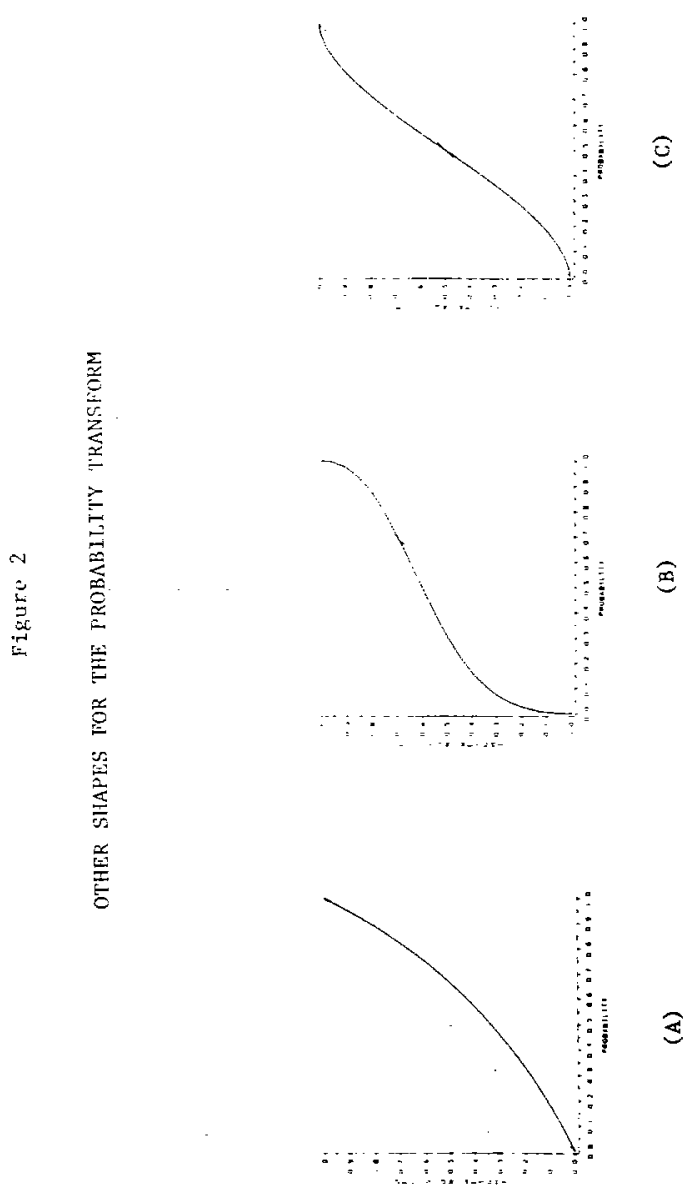
Table 1

Shape and Interpretation of the Outcome Transform

\begin{tabular}{|c|c|c|c|c|}
\hline \multirow[b]{2}{*}{ Value of $\gamma$} & \multirow[b]{2}{*}{$\begin{array}{l}\text { Shape } \\
\text { of the } \\
\text { Outcome } \\
\text { Transform }\end{array}$} & \multicolumn{3}{|c|}{ Interpretation } \\
\hline & & $\begin{array}{c}\text { Expected Utility/ } \\
\text { Subjective Expected } \\
\text { Utility (Savage) model }\end{array}$ & $\begin{array}{l}\text { Probability } \\
\text { Transform }\end{array}$ & $\begin{array}{l}\text { Prospect } \\
\text { Theory }\end{array}$ \\
\hline$y<1$ & Concave & Risk Aversion & & $\begin{array}{l}\text { Assumed } \\
\text { Over Gains }\end{array}$ \\
\hline$\gamma=1$ & Linear & $\begin{array}{c}\text { Risk Neutrality } \\
\text { (Expected Value Model) }\end{array}$ & $\begin{array}{l}\text { Assumed } \\
\text { Linear }\end{array}$ & \\
\hline$y>1$ & Convex & Risk Seeking & & $\begin{array}{c}\text { Assumed } \\
\text { Over Losses }\end{array}$ \\
\hline
\end{tabular}


Table 2

Implications for the Decision-Making Models

Mode1

Percent of Subjects, Gains

Percent of Subjects, Losses

Explicitly considered

Expected Value

10.5

10.5
Expected Utility
(risk aversion)
Expected Utility
(risk seeking)

5.3

0.0

0.0

5.3

Subjective Expected Utility

(risk aversion)

10.5

0.0

Subjective Expected Utility

5.3

3.5

(risk seeking)

Probability Transform

29.8

22.8

(SEU-type)

Probability Transform

(PT-type)

12.3

28.1

Prospect Theory (PT)

7.0

$1.8^{\mathrm{a}}$

(concave value function)

Prospect Theory

(convex value function)

0.0

$12 \cdot 3^{a}$

\section{"Aberrent"}

Supracertain

10.5

10.5

Always Underweight

Probabilities

7. 0

3.5

Mirror Subjective Expected

Utility

1.8

1.8

a Since we treated losses as positive numbers for purposes of estimation, an estimated concave function $(\gamma<1)$ equates to a convex function in the loss domain (negative quadrant) and an estimated convex function to a concave one. For the purposes of this table, we have classified results as they would be if the function were estimated in the loss domain. 